\title{
ANÁLISIS COMPARATIVO DE LA FASE SEXUAL DE DOS ESPECIES DE MICROGRAMMA (POLYPODIACEAE, PLEOPELTOIDEAE)
}

\author{
Aniceto Mendoza-Ruiz y Blanca Pérez-García \\ Área de Botánica Estructural y Sistemática Vegetal, Depto. de Biología, \\ Universidad Autónoma Metropolitana, Iztapalapa. Apdo. postal 55-535, \\ 09340 México, D.F. e-mail: amr@xanum.mx
}

\section{RESUMEN}

Se describe la morfología de los gametofitos de Microgramma lycopodioides y Microgramma piloselloides. Ambas especies presentan esporas monoletes, germinación de tipo Vittaria y desarrollo protálico de tipo Drynaria. Sus gametofitos son cordiformeespatulados a cordiforme-reniformes, con desarrollo de escasos pelos unicelulares y pluricelulares en la etapa adulta. Las primeras hojas del esporofito son simples y espatuladas con estomas polocíticos. Se compara y discute el desarrollo protálico de ambas plantas con el de Microgramma nitida y de algunas especies del género Pleopeltis, observando que todas ellas comparten el mismo tipo de germinación tipo Vittaria y el desarrollo protálico tipo Drynaria.

Palabras clave: gametofito, morfogénesis, Microgramma, Pleopeltoideae, Polypodiaceae.

\section{ABSTRACT}

A comparison was made of the gametophytic development of Microgramma lycopodioides and Microgramma piloselloides. Both species present monolete spores, Vittaria-type germination and Drynaria-type prothallial development. Adult gametophytes are cordate-spatulate to cordate-reniform, with the development of scarce unicellular and pluricellular hairs in the adult stage. The first leaves of the sporophyte are simple and spatulate with polocytic stomata. A comparison was also made and discussed between the prothallic development of both species and that of Microgramma nitida as well as of some species of the genus Pleopeltis, which also present Vittaria-type germination and Drynaria-type prothallial development.

Key words: gametophyte, morphogenesis, Microgramma, Pleopeltoideae, Polypodiaceae. 


\section{INTRODUCCIÓN}

Microgramma C. Presl es un género que incluye aproximadamente 24 especies de distribución neotropical y junto con Pleopeltis Humb. et Bonpl. ex Willd. se ubica dentro de la subfamilia Pleopeltoideae, familia Polypodiaceae (Mickel y Beitel, 1988). Las especies de Microgramma crecen desde el nivel del mar hasta los 2200 m s.n.m. y las de Pleopeltis las encontramos creciendo desde 150 hasta los 3100 m s.n.m. (Mickel y Smith, 2004), principalmente en las partes abiertas de las selvas altas perennifolias y bosques templados de Quercus. Los representantes de Microgramma se caracterizan por ser plantas epifitas o raramente rupícolas con el rizoma largamente rastrero y escamoso, con las hojas simples, que varían de monomorfas a marcadamente dimorfas, presentan esporas monoletes, con el perisporio granulado a reticulado y de color amarillo (Moran, 1995). Las especies de Microgramma difieren de las de Pleopeltis por presentar las escamas del rizoma no clatradas y los parafisos generalmente no peltados.

Hasta el momento se han realizado estudios sobre la morfogénesis de la fase sexual de Microgramma heterophylla (L.) Wherry (Chiou y Farrar, 1997), Microgramma nitida (J. Sm.) A. R. Sm. (Ramírez Trejo y Pérez-García, 1998) y algunas especies de Pleopeltis (Bhattacharyya y Sen, 1992; Reyes Jaramillo et al., 2003; Nayar, 1962). Para ambos casos se ha encontrado que el patrón de germinación es de tipo Vittaria y el desarrollo protálico de tipo Drynaria (Nayar y Kaur, 1969, 1971).

Con el fin de contribuir a un mejor conocimiento y comprensión de la morfogénesis de la fase sexual en la subfamilia Pleopeltoidae, en este trabajo se describe la morfología de los gametofitos de Microgramma lycopodioides (L.) Copel. y Microgramma piloselloides (L.) Copel. y se le compara con los resultados obtenidos por otros autores para este grupo de helechos (Bhattacharyya y Sen, 1992; Chiou y Farrar, 1997; Nayar, 1962; Ramírez Trejo y Pérez-García, 1998; Reyes Jaramillo et al., 2003).

\section{MATERIALES Y MÉTODOS}

Las hojas fértiles de Microgramma lycopodioides y Microgramma piloselloides fueron recolectadas en la localidad de La Gran Piedra, provincia de Santiago de Cuba, Cuba (A. Mendoza, s. n.), en bosque mesófilo de montaña a $800 \mathrm{~m}$ s.n.m. También se obtuvo material de $M$. lycopodioides recolectada en la localidad de Jesús Carranza, mpio. de Hidalgotitlán, estado de Veracruz, México (I. Reyes, 172), en restos de selva alta perennifolia a $300 \mathrm{~m}$ s.n.m. Los ejemplares

de respaldo se encuentran depositados en los herbarios BSC (Biodiversidad Santiago 
de Cuba) del Jardín de los Helechos de Santiago de Cuba y en el Herbario Metropolitano UAMIZ de la Universidad Autónoma Metropolitana, Iztapalapa.

Las pinnas fértiles se guardaron en sobres de papel con el fin de que en su interior se liberaran las esporas. Posteriormente se tamizó el contenido del sobre con una malla metálica con poros de $0.074 \mathrm{~mm}$ de diámetro, para eliminar restos de esporangios e indusios. Las esporas obtenidas se sembraron en cajas de Petri de $5 \mathrm{~cm}$ de diámetro (20 cajas por cada especie), que contenían agar y medio de cultivo de Thompson previamente esterilizado (Klekowski, 1969), esparciéndolas con un pincel de pocas cerdas sobre la superficie del medio solidificado, a una densidad promedio de 100-150 esporas por $\mathrm{cm}^{2}$. De igual forma se sembraron esporas de cada especie en pequeñas macetas con tierra.

Las cajas y las macetas se mantuvieron dentro de bolsas de plástico transparente para evitar contaminación y desecación y con un fotoperiodo de $12 \mathrm{hrs}$ luz/oscuridad, con luz artificial (lámparas de 75 watts, luz de día), a una temperatura de 20 a $25{ }^{\circ} \mathrm{C}$.

Se hizo el seguimiento de cada una de las fases del ciclo sexual de ambas especies, realizando observaciones dos veces por semana durante más de un año, tomando fotomicrografías de cada una de las distintas etapas.

\section{RESULTADOS}

Las esporas en ambas especies son monoletes, elipsoidales, con el perisporio granulado a tuberculado, de color amarillo brillante, las de Microgramma lycopodioides miden 48 (51) 55 X 34 (35) $40 \mu \mathrm{m}$ y las de M. piloselloides 55 (61) 70 X 38 (43) $50 \mu \mathrm{m}$ (Figs. 1, 2).

En los dos taxa las esporas comienzan a germinar entre los 7 y los 10 días posteriores a la siembra; el patrón de germinación que presentan es de tipo Vittaria, en el cual la primera célula rizoidal se forma a partir de una primera división celular perpendicular al eje polar de la espora (Fig. 3); la formación de un filamento germinativo se inicia a partir de una segunda división que es perpendicular a la primera.

El desarrollo protálico en ambas especies es de tipo Drynaria, caracterizado por el establecimiento retardado de la célula meristemática. En este patrón y como resultado de las divisiones celulares, se forman filamentos delgados y uniseriados de 2 a 8 células de longitud (Fig. 4), que se desarrollan entre los 10 y los 15 días, para dar luego paso a la estructuración de una lámina protálica que comienza su desarrollo entre los 15 y los 26 días, iniciando su integración a partir de las células apicales del filamento, por divisiones longitudinales y transversales. Cuando el gametofito adquiere una forma laminar ovada, se diferencia una célula meristemática 

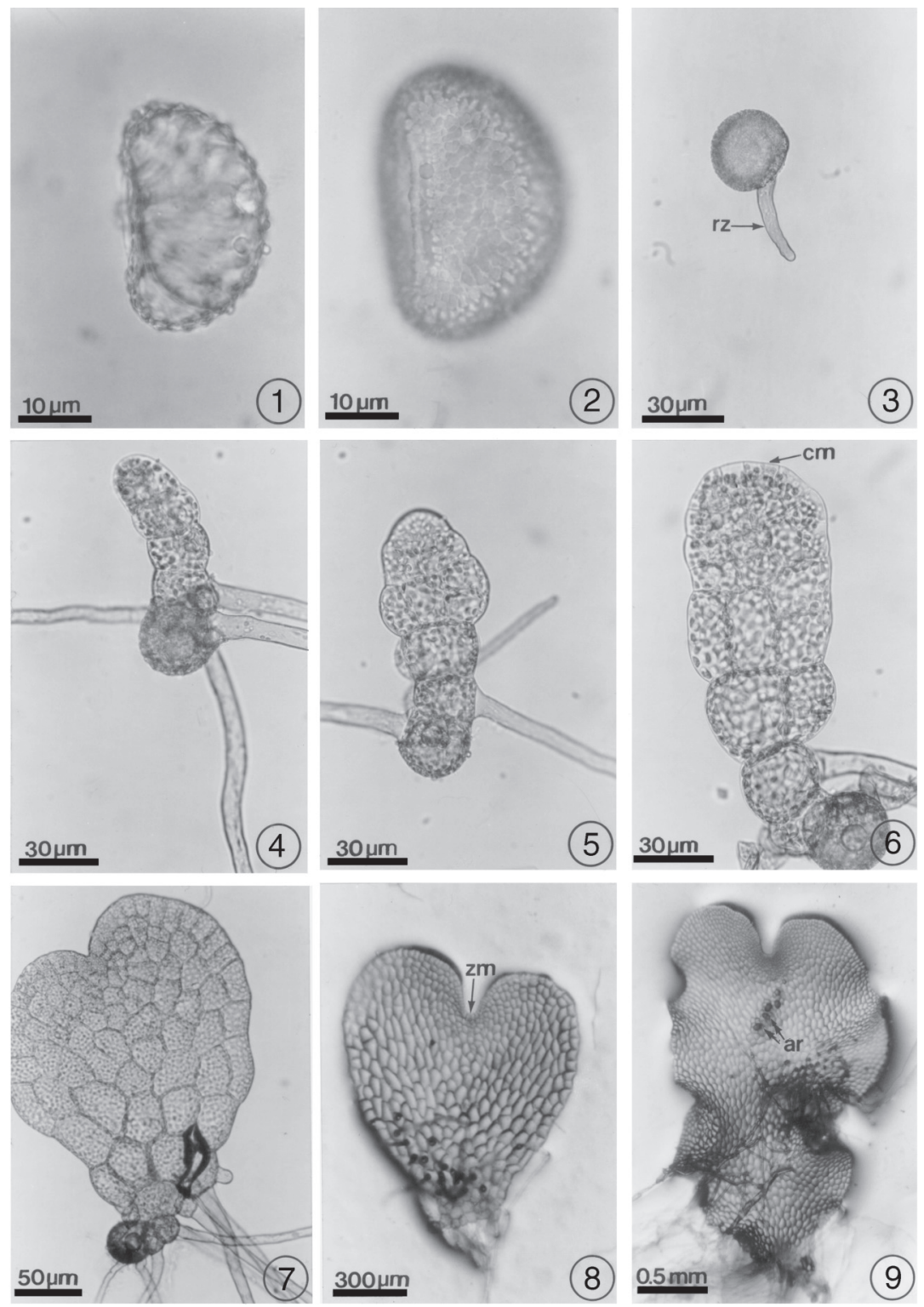

Figs.1-9. Morfología de los gametofitos de Microgramma lycopodioides y Microgramma piloselloides. 1. Espora de M. lycopodioides; 2. espora de M. piloselloides; 3. germinación, M. piloselloides (7 días); 4-5. filamento de cinco células e inicio de la formación de la lámina, M. lycopodioides (15 días); 6. lámina joven, M. piloselloides (20 días); 7-8. fases laminares, M. lycopodioides (26 y 42 días); 9. gametofito adulto con arquegonios, M. piloselloides (75 días). ar= arquegonios, $\mathrm{cm}=$ célula meristemática, $\mathrm{rz}=$ rizoide, $\mathrm{zm}=$ zona meristemática. 
que más tarde es reemplazada por un meristemo pluricelular y se desarrolla un cojinete muy delgado; en esta primera etapa (26-45 días) los gametofitos son desnudos (Figs. 5-7).

Los gametofitos adultos son cordiforme-espatulados a cordiforme-reniformes y en esta fase se desarrollan escasos pelos unicelulares en el margen de la lámina y algunos pelos bi-tricelulares entre la zona meristemática y el cojinete (60-100 días, Figs. 9-13). El cojinete está poco diferenciado y los gametangios aparecen cerca de la zona meristemática correspondiendo al tipo común de los helechos leptosporangiados (Fig. 9).

Los arquegonios, desarrollados entre los 60 y los 100 días, están formados por cuatro hileras de células, cada una con 4 o 5 células de longitud (Figs. 9, 14). Por su parte los anteridios se originan entre los 100 y los 150 días, son globosos y están constituidos por una célula basal, una célula media y una célula opercular (Fig.15). Aunque se encontraron gametofitos unisexuados, no se observaron evidencias de efecto anteridiogénico, pues los gametofitos se desarrollan de manera normal.

Las primeras hojas del esporofito se observaron después de los 120 días de cultivo y, en ambas especies, la lámina es simple, espatulada, corta, con pelos unicelulares a tricelulares bifurcados y en la cara abaxial de la lámina se observan estomas de tipo polocítico (Van Cotthem, 1970a) que se caracterizan por presentar una célula subsidiaria rodeada por células epidérmicas de paredes onduladas (Figs. 16-17). Cabe enfatizar que los diversos tipos estomáticos en cuanto a su morfología y ontogenia, se han considerado como un carácter importante en la taxonomía de las plantas (Thurston, 1969; Van Cothem, 1970a, 1970b).

\section{DISCUSIÓN}

La observación de la morfología de los gametofitos de Microgramma lycopodioides y M. piloselloides nos permite concluir que ambas especies comparten las siguientes características: a) esporas monoletes y elipsoidales de color amarillo; b) germinación de tipo Vittaria y c) desarrollo protálico de tipo Drynaria y gametofitos adultos con escasos pelos unicelulares marginales. Al comparar nuestros resultados con los dados a conocer por Ramírez Trejo y Pérez-García (1998) para los gametofitos de Microgramma nitida (J. Sm.) A. R. Sm., los obtenidos por Chiou y Farrar (1997) para M. heterophylla (L.) Wherry y los observados para Pleopeltis astrolepis, P. crassinervata, P. macrocarpa, P. polylepis y P. revoluta por Reyes Jaramillo et al. (2003), podemos señalar la presencia de un patrón de germinación de tipo Vittaria y de un desarrollo protálico de tipo Drynaria, común dentro de la subfamilia Pleopeltoideae (Cuadro 1). Sin embargo, cabe indicar que 

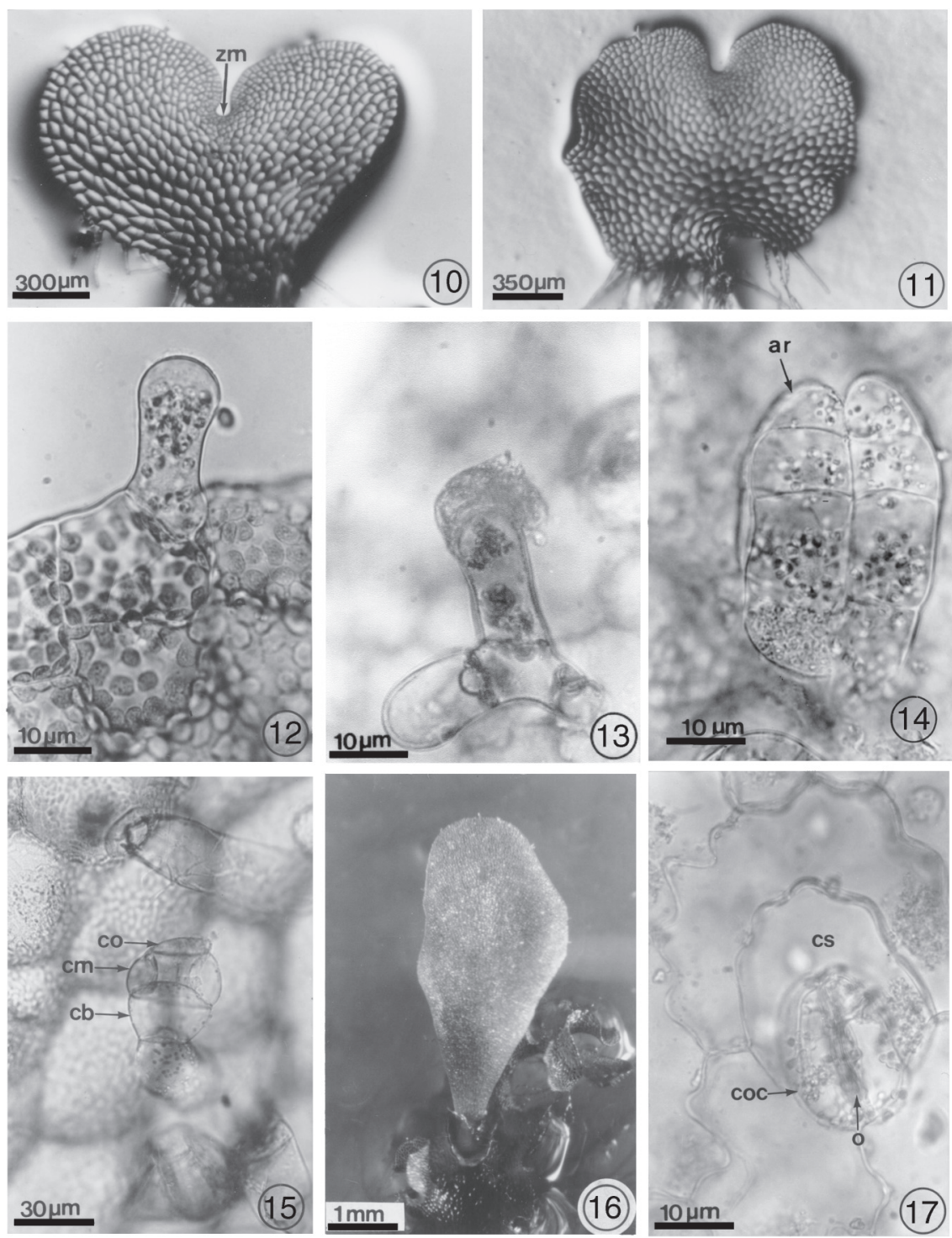

Figs. 10-17. Morfología de los gametofitos de Microgramma lycopodioides y Microgramma piloselloides. 10. gametofito cordiforme, M. lycopodioides (67 días); 11. gametofito cordiforme, M. piloselloides (60 días); 12. pelo unicelular marginal, M. piloselloides; 13. pelo tricelular superficial, M. piloselloides; 14. arquegonio, M. piloselloides (75 días); 15. anteridios, $M$. lycopodioides (99 días); 16-17. primera hoja del esporofito y estoma polocítico, M. piloselloides. $\mathrm{an}=$ anteridios, $\mathrm{ar}=$ arquegonios, $\mathrm{cb}=$ célula basal, $\mathrm{cm}=$ célula media, $\mathrm{co}=$ célula opercular, $\mathrm{coc}=$ célula oclusiva, $\mathrm{cs}=$ célula subsidiaria, $\mathrm{o}=$ ostiolo, $\mathrm{zm}=$ zona meristemática. 
Cuadro 1. Comparación del desarrollo protálico de Microgramma lycopodioides y M. piloselloides con el de otras especies de la subfamilia Pleopeltoideae.

\begin{tabular}{|c|c|c|c|c|c|c|}
\hline $\begin{array}{c}\text { Taxa } \\
\text { Caracteres }\end{array}$ & $\begin{array}{l}\text { Microgramma } \\
\text { lycopodioides }\end{array}$ & $\begin{array}{l}\text { Microgramma } \\
\text { piloselloides }\end{array}$ & $\begin{array}{c}{ }^{1} \text { Microgramma } \\
\text { heterophylla }\end{array}$ & ${ }^{2}$ Microgramma nitida & $\begin{array}{c}{ }^{3} \text { Pleopeltis astrolepis, } \\
\text { P. crassinervata, } P . \text { polylepis } \\
\text { y } P . \text { revoluta }\end{array}$ & $\begin{array}{c}\text { Pleopeltis angusta y } \\
\text { P. mexicana }\end{array}$ \\
\hline $\begin{array}{l}\text { Esporas } \\
\text { monoletes }\end{array}$ & $\begin{array}{l}48(51) 55 \times 34(35) 40 \\
\mu \mathrm{m} \text {, perisporio } \\
\text { escasamente tuberculado } \\
\text { a granulado }\end{array}$ & $\begin{array}{l}55(61) 70 \times 38(43) 50 \\
\mu \mathrm{m}, \text { perisporio } \\
\text { tuberculado a granulado }\end{array}$ & & $\begin{array}{l}35 \times 20 \mu \mathrm{m} \text {, perisporio } \\
\text { tuberculado a granulado }\end{array}$ & $\begin{array}{l}42(45) 50 \times 30(33) 35 \mu \mathrm{m}, \\
\text { perisporio liso a ligeramente } \\
\text { tuberculado }\end{array}$ & $\begin{array}{l}60(76) 88 \times 36(54) 60 \\
\mu \mathrm{m}, \text { perisporio liso a } \\
\text { ligeramente tuberculado }\end{array}$ \\
\hline Germinación & tipo Vittaria & tipo Vittaria & tipo Vittaria & tipo Vittaria & tipo Vittaria & tipo Pleopeltis \\
\hline $\begin{array}{l}\text { Fase } \\
\text { filamentosa }\end{array}$ & $\begin{array}{l}\text { filamentos de } 2 \text { a } 6 \\
\text { células }\end{array}$ & $\begin{array}{l}\text { filamentos de } 2 \text { a } 8 \\
\text { células }\end{array}$ & $\begin{array}{l}\text { filamentos de } 2 \text { a } 8 \\
\text { células }\end{array}$ & filamentos de 6 células & $\begin{array}{l}\text { filamentos de } 2 \text { a } 5 \text { células, } \\
\text { raramente de } 7 \text { células }\end{array}$ & $\begin{array}{l}\text { filamentos de } 2 \text { a } 5 \\
\text { células }\end{array}$ \\
\hline $\begin{array}{l}\text { Desarrollo } \\
\text { protálico }\end{array}$ & tipo Drynaria & tipo Drynaria & $\begin{array}{l}\text { tipo Drynaria o } \\
\text { tipo Aspidium }\end{array}$ & tipo Drynaria & tipo Drynaria & tipo Ceratopteris \\
\hline Fase adulta & $\begin{array}{l}\text { cordiforme-espatulados a } \\
\text { cordiforme-reniformes }\end{array}$ & $\begin{array}{l}\text { cordiforme-espatulados a } \\
\text { cordiforme-reniformes }\end{array}$ & $\begin{array}{l}\text { cordiformes o en forma } \\
\text { de banda con clones }\end{array}$ & $\begin{array}{l}\text { cordiformes alargados, } \\
\text { márgenes ondulados }\end{array}$ & $\begin{array}{l}\text { cordiforme-espatulados a } \\
\text { cordiforme-reniformes }\end{array}$ & $\begin{array}{l}\text { cordiforme-espatulados a } \\
\text { cordiforme-reniformes }\end{array}$ \\
\hline Pelos & unicelulares capitados & $\begin{array}{l}\text { unicelulares capitados, } \\
\text { tricelulares ramificados }\end{array}$ & $\begin{array}{l}\text { unicelulares capitados, } \\
\text { tricelulares ramificados }\end{array}$ & unicelulares capitados & unicelulares capitados & unicelulares capitados \\
\hline Gametangios & $\begin{array}{l}\text { O se forman a partir de } \\
80-100 \text { días. } \\
9 \text { se forman a partir de } \\
100-200 \text { días, tienen } 3 \\
\text { células: una basal, una } \\
\text { media y una opercular }\end{array}$ & $\begin{array}{l}\text { O se forman a partir de } \\
60-100 \text { días. } \\
\text { q se forman a partir de } \\
100 \text { días, tienen } 3 \text { células: } \\
\text { una basal, una media y } \\
\text { una opercular }\end{array}$ & $\begin{array}{l}\text { Ôse forman a partir de } \\
60-100 \text { días. } \\
\text { O se forman a partir de } \\
100 \text { días, tienen } 3 \text { células: } \\
\text { una basal, una media y } \\
\text { una opercular }\end{array}$ & $\begin{array}{l}\text { T se forman a partir de } 90 \\
\text { días. } \\
\text { } 9 \text { se forman a partir de } \\
100 \text { días, tienen } 3 \text { células: } \\
\text { una basal, una media y } \\
\text { una opercular }\end{array}$ & $\begin{array}{l}\text { O se forman a partir de } 40- \\
130 \text { días. } \\
9 \text { se forman a partir de } 50 \text { - } \\
100 \text { días, tienen } 3 \text { células: } \\
\text { una basal, una media y una } \\
\text { opercular }\end{array}$ & $\begin{array}{l}\text { O se forman a partir de } \\
40-130 \text { días. } \\
0 \text { se forman a partir de } \\
50-130 \text { días, tienen } 3 \\
\text { células: una basal, una } \\
\text { media y una opercular }\end{array}$ \\
\hline Esporofito & $\begin{array}{l}\text { no se formaron } \\
\text { esporofitos después de } \\
300 \text { días de cultivo }\end{array}$ & $\begin{array}{l}\text { las primeras hojas se } \\
\text { forman después de los } 4 \\
\text { meses, lámina simple con } \\
\text { pelos unicelulares }\end{array}$ & & $\begin{array}{l}\text { las primeras hojas se } \\
\text { forman después de } 8 \\
\text { meses, lámina simple } \\
\text { espatulada con pelos } \\
\text { pluricelulares }\end{array}$ & $\begin{array}{l}\text { no se formaron esporofitos } \\
\text { después de } 300 \text { días de } \\
\text { cultivo }\end{array}$ & $\begin{array}{l}\text { las primeras hojas son } \\
\text { simples, espatulado- } \\
\text { alargadas con pelos } \\
\text { unicelulares }\end{array}$ \\
\hline Estomas & & $\begin{array}{l}\text { polocíticos, con una } \\
\text { célula subsidiaria }\end{array}$ & & anomocíticos & & anomocíticos \\
\hline
\end{tabular}

${ }^{1}$ Chiou y Farrar, 1997; ${ }^{2}$ Ramírez Trejo y Pérez-García, 1998; ${ }^{3}$ Reyes Jaramillo et al., 2003. 
M. heterophylla puede presentar ocasionalmente un desarrollo protálico de tipo Aspidium, lo que hasta el momento no se ha observado en otros taxa de la subfamilia. Por otra parte Pleopeltis angusta y P. mexicana se diferencian del resto de las especies porque presentan una germinación de tipo Pleopeltis y un desarrollo protálico de tipo Ceratopteris (Reyes Jaramillo et al., 2003).

En lo que se refiere al tamaño de las esporas, las de M. lycopodioides (51 x $35 \mu \mathrm{m})$ y de $M$. piloselloides $(61$ x $43 \mu \mathrm{m})$ son más grandes que las de $M$. nitida (35 x $20 \mu \mathrm{m}$, Ramírez Trejo y Pérez-García, 1998). Comparando estos datos con los definidos para Pleopeltis astrolepis, P. crassinervata, P. macrocarpa, P. polylepis y $P$. revoluta, cuyas esporas miden en promedio 45 x $33 \mu \mathrm{m}$, se observa que estas últimas son ligeramente más pequeñas. A diferencia de todas las especies anteriores, las esporas de $P$. angusta y $P$. mexicana son mucho más grandes (76 x $54 \mu \mathrm{m})$. Es interesante notar que la variación en el tamaño de las esporas de los helechos mencionados (Cuadro 1) sugiere la existencia de distintos niveles de ploidía en este grupo de plantas (Tryon y Lugardon, 1991).

Podemos decir que en las especies de la familia Polypodiaceae estudiadas hasta ahora se presenta un patrón de desarrollo protálico de tipo Drynaria; lo que aunado a la presencia de un glóbulo de grasa en la primera célula protálica, así como a la formación de escasos pelos unicelulares capitados en los márgenes y ocasionalmente en la superficie de la lámina en etapas adultas de los gametofitos, y a la formación asincrónica de los gametangios característicos de los helechos leptosporangiados, define caracteres que pueden considerarse como diagnósticos para este grupo de plantas (Chiou y Farrar, 1997; Reyes y Pérez-García, 1994; Reyes et al. 1996).

Cabe mencionar que los gametofitos estudiados son unisexuales, lo cual facilita el entrecruzamiento intergametofítico y aunque la formación de los gametangios es asincrónica, no hay presencia de anteridiógenos en estas especies, ya que la función de las mencionadas hormonas consiste en inducir la formación precoz de anteridios en gametofitos filamentosos de corta edad, hecho que no se ha observado. Los anteridiógenos operan en la naturaleza causando alteración en la biología reproductiva de los gametofitos de los helechos, trayendo como consecuencia un aumento de la variabilidad genética (Raghavan, 1989).

Finalmente las diversas descripciones morfológicas de los gametofitos hechas por varios autores (Atkinson,1973; Atkinson y Stokey, 1964; Nayar y Kaur, 1971) han puesto de manifiesto que éstas incluyen datos útiles en la diferenciación de los taxa de helechos a distintos niveles, como por ejemplo las familias Grammitidaceae y Schizaeaceae o los géneros Davallia, Nephrolepis y algunos otros (Atkinson, 1973). Lo anterior sugiere que la revisión de los caracteres de los gametofitos, aunada a la de los esporofitos debe ser considerada en la sistemática de las pteridofitas. 
Mendoza-Ruiz y Pérez-García: Análisis de la fase sexual de dos especies de Microgramma

\section{AGRADECIMIENTOS}

Los autores agradecen a Jacqueline Ceja y a los revisores por sus comentarios y valiosas sugerencias al manuscrito, a Jorge Lodigiani por el apoyo fotográfico.

\section{LITERATURA CITADA}

Atkinson, L. R. 1973. The gametophytes and family relationships. In: Jermy, A. C., J. A. Crabbe, y B. A. Thomas (eds.). The phylogeny and classification of the ferns. Bot. J. Linn. Soc. 67 (Suppl. 1): 73-90.

Atkinson, L. R. y A. G. Stokey. 1964. Comparative morphology of the gametophyte of homosporous ferns. Phytomorphology 14: 51-70.

Bhattacharyya, A. K. y T. Sen. 1992. Structure and ontogeny of gametophytes of Lepisorus (J. Sm.) Ching and Pleopeltis Willd. Indian Fern J. 9: 66-75.

Chiou, W. L. y D. R. Farrar. 1997. Comparative gametophyte morphology of selected species of the family Polypodiaceae. Amer. Fern J. 87: 77-86.

Klekowski, E. J. Jr. 1969. Reproductive biology of the Pteridophyta. III. A study of the Blechnaceae. J. Linn. Soc. 62: 361-377.

Mickel, J. T. y J. M. Beitel. 1988. Microgramma. In: Pteridophyte flora of Oaxaca, Mexico. Mem. New York Bot. Gard. 46: 249-251.

Mickel, J. T. y A. R. Smith. 2004. The pteridophytes of Mexico. Mem. New York Bot. Gard. 88: 1-1054.

Moran, R. C. 1995. Polypodiaceae. In: Moran, R. C. y R. Riba (eds.). Flora Mesoamericana Vol. 1. Psilotaceae a Salviniaceae. Instituto de Biología, Universidad Nacional Autónoma de México. México, D.F. pp. 333-366.

Nayar, B. K. 1962. Morphology of spores and prothalli of some species of Polypodiaceae Bot. Gaz. (Crawfordsville) 123: 223-232.

Nayar, B. K y S. Kaur. 1969. Types of prothallial development in homosporous ferns. Phytomorphology 19: 179-188.

Nayar, B.K. y S. Kaur. 1971. Gametophytes of homosporous ferns. Bot. Rev. (Lancaster) 37: 295-396.

Raghavan, V. 1989. Developmental biology of ferns gametophytes. Cambridge University Press. Nueva York. 361 pp.

Ramírez Trejo, M. R. y B. Pérez-García. 1998. Fase gametofítica de Microgramma nitida (Polypodiaceae). Rev. Biol. Trop. 46: 587-593.

Reyes, J. I. y B. Pérez-García. 1994. Morfología y estrategias reproductivas del gametofito de Polypodium lepidotrichum (Fée) Maxon (Polypodiaceae). Acta Bot. Mex. 28: 7178.

Reyes, J. I., B. Pérez-García y A. Mendoza. 1996. Desarrollo del gametofito y del esporofito joven de Niphidium crassifolium (Filicales: Polypodiaceae s. str.). Rev. Biol. Trop. 44: 485-490. 
Reyes Jaramillo, I., B. Pérez-García y A. Mendoza Ruiz. 2003. Morfogénsis de los gametofitos de especies mexicanas de Pleopeltis (Polypodiaceae, subfamilia Pleopeltoideae). Rev. Biol. Trop. 51:321-332.

Thurston, E. L. 1969. Taxonomic significance of stomatal patterns in the ferns. Amer. Fern J. 59: 68-79.

Tryon, A. F. y B. Lugardon. 1991. Spores of the Pteridophyta: surface, wall structures and diversity based on electron microscope studies. Springer-Verlag. Nueva York. 648 pp.

Van Cotthem, W. R. J. 1970a. A classification of stomatal types. Bot. J. Linn. Soc. 63: 235-246.

Van Cotthem, W. R. J. 1970b. Comparative morphological study of the stomata in the Filicopsida. Bull. Jard. Bot. Etat Belg. 40: 81-239. 